\title{
Towards the GEOSAT Follow-On Precise Orbit Determination Goals of High Accuracy and Near-Real-Time Processing
}

\author{
Frank G. Lemoine* \\ Planetary Geodynamics Laboratory, NASA GSFC, Greenbelt, Maryland USA \\ Nikita P. Zelensky ${ }^{\dagger}$ \\ SGT Inc., Greenbelt, Maryland USA \\ Douglas S. Chinn \\ SGT Inc., Greenbelt, Maryland USA \\ Brian D. Beckley $\S$ \\ SGT Inc., Greenbelt, Maryland, USA \\ John L. Lillibridge I \\ Laboratory for Satellite Altimetry, NOAA, Silver Spring, Maryland, USA
}

\begin{abstract}
The US Navy's GEOSAT Follow-On spacecraft (GFO) primary mission objective is to map the oceans using a radar altimeter. Satellite laser ranging data, especially in combination with altimeter crossover data, offer the only means of determining high-quality precise orbits. Two tuned gravity models, PGS7727 and PGS7777b, were created at NASA GSFC for GFO that reduce the predicted radial orbit hrough degree 70 to 13.7 and $10.0 \mathrm{~mm}$. A macromodel was developed to model the nonconservative forces and the SLR spacecraft measurement offset was adjusted to remove a mean bias. Using these improved models, satellite-ranging data, altimeter crossover data, and Doppler data are used to compute both daily medium precision orbits with a latency of less than 24 hours. Final precise orbits are also computed using these tracking data and exported with a latency of three to four weeks to NOAA for use on the GFO Geophysical Data Records (GDR's). The estimated orbit precision of the daily orbits is between 10 and $20 \mathrm{~cm}$, whereas the precise orbits have a precision of $5 \mathrm{~cm}$.
\end{abstract}

\section{Nomenclature}

Subscripts

$l \quad$ spherical harmonic degree

$m \quad$ spherical harmonic order

Conventions

$\bar{C}_{l m}, \bar{S}_{l m}$ Normalized spherical harmonic coefficients

$\bar{P}_{l m} \quad$ Normalized associated Legendre function

$c_{d} \quad$ Drag coefficient

$G M \quad$ Gravitational constant, $\mathrm{m}^{3} / \mathrm{s}^{2}$

${ }^{*}$ Research Scientist, Planetary Geodynamics Laboratory, Code 698, NASA Goddard Space Flight Center, Greenbelt, Mary* land, 20771 USA, Member.

†Principal Analyst, SGT Inc., 7701 Greenbelt Road, Greenbelt, Maryland 20770 USA.

$\ddagger$ Principal Programmer/Analyst, SGT Inc., 7701 Greenbelt Road, Greenbelt, Maryland 20770 USA.

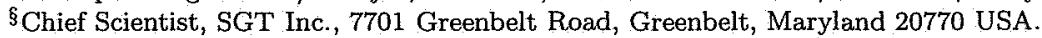

Research Scientist, Laboratory for Satellite Altimetry, SSMC-I, E/RA 31, 1335 East-West Highway, Silver Spring, Maryland 20910 USA

This material is declared a work of the U.S. Government and is not subject to copyright protection in the United States.2006 


\begin{tabular}{ll}
$k_{2}$ & Love number of degree two \\
$k_{3}$ & Love number of degree three \\
$r$ & radius \\
$R_{e}$ & Reference radius for gravity model \\
$U$ & Gravity potential \\
CDDIS & Crustal Dynamics Data Information System \\
DGFI & Deutsches Geodätisches Forschungsinstitut \\
GFO & Geosat-Follow-On \\
GOT00.2 Goddard Ocean Tide Model 2000, Version 2 \\
GPS & Global Positioning System \\
IGDR & Intermediate Geophysical Data Record \\
ILRS & International Laser Ranging Service \\
IRI & International Reference Ionosphere \\
LRA & Laser Retroreflector Array \\
MOE & Medium precision Orbit Ephemerides \\
NCEP & National Center for Environmental Prediction \\
opr & once-per-revolution \\
POD & Precision orbit determination \\
POE & Precise Orbit Ephemerides \\
SLR & Satellite Laser Ranging \\
SWH & Significant Wave Height \\
T/P & TOPEX/Poseidon \\
WVR & Water Vapour Radiometer \\
Symbols \\
$\beta$ & Specular reflectivity \\
$\delta$ & Diffuse reflectivity \\
$\lambda$ & longitude \\
$\phi$ & latitude \\
\hline
\end{tabular}

\section{Introduction}

\section{I.A. Background}

The GEOSAT Follow-On (GFO) spacecraft was launched on February 10, 1998 with the objective of providing continuous ocean observations along the GEOSAT exact repeat ground track for both real-time and nearreal-time measurements of relative ocean heights, and absolute heights for large-scale ocean modelling. The inclination and ground-track repeat period (17 days) complement the data collected by other missions, such as TOPEX/Poseidon, ERS-1, ERS-2, ENVISAT and Jason-1 (see Table 1).

GFO carries a single-frequency $(13.5 \mathrm{Ghz})$ altimeter, a dual-

Table 1. GFO Orbit Summary

\begin{tabular}{lc}
\hline Altitude & $800 \mathrm{~km}$ \\
Eccentricity & 0.008 \\
Inclination & $108^{\circ}$ \\
Repeat Period & 244 revs in 17 days \\
\hline
\end{tabular}
frequency water vapour radiometer, a dual-frequency Doppler (TRANET-style) beacon for operational tracking, and a laser retro reflector array (LRA) for precision orbit determination (see Fig. 1). The satellite also carried GPS dual-frequency receivers, however the GPS system on GFO only supplied limited data, and could not be used for precision orbit determination (POD). Hence, both the operational and precise orbits have been determined using a combination of satellite laser ranging (SLR) and Doppler tracking in combination with the use of altimeter crossovers. The spacecraft was declared operational on November 29, 2000, and has now returned nearly six years of altimeter data over 120 repeat cycles. GFO has occupied the GEOSAT Exact Repeat Orbit and supplied useful data longer than the original GEOSAT mission which provided data in this orbit from 1986 to $1989.1,2$

The GFO spacecraft was constructed by Ball Aerospace and launched on a Taurus launch vehicle from Vandenburg Air Force Base on February 10, 1998 for the prime customer, the U.S. Navy. The National Oceanic and Atmospheric Administration has the responsibility for distribution of the altimeter data. The Planetary Geodynamics Branch at the NASA Goddard Space Flight Center produces the operational and precise orbits for GFO. The precise orbits are supplied to NOAA for placement on the Geophysical Data 
Records (GDR's) which are released to the scientific community.

\section{I.B. Orbit Determination Challenge}

For altimeter satellites, the prime observation is the range measurement from the satellite to the ocean surface. It follows that the ability to discriminate changes in the height (or topography) of the ocean depends on the on knowledge of the satellite orbit, and that the satellite orbit must be known as accurately as possible. The precision of the orbit depends on the quality of the tracking data, the fidelity of the force and measurement models, and the choice of parameters estimated in an orbit determination solution. It is the knowledge of the radial component of the orbit that is the most critical for satellite altimetry. Over the 13 years of its mission, $\mathrm{T} / \mathrm{P}$ achieved radial orbit precision of 2-2.5 cm. ${ }^{3,4}$ For the successor mission, Jason-1, orbits with a radial precision of $1-\mathrm{cm}$ have been achieved. ${ }^{4,5}$ Both $\mathrm{T} / \mathrm{P}$ and Jason-1 benefited directly from the near-continuous tracking supplied by DORIS (Doppler Orbitography and Radio

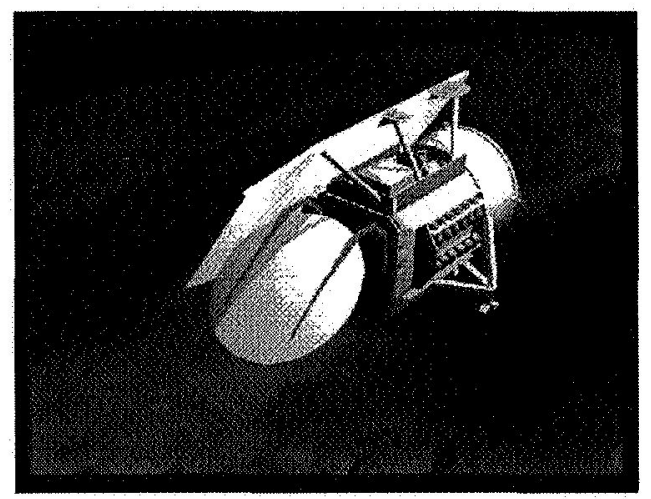

Figure 1. Geosat Follow-On Positioning Integrated by Satellite) or GPS (Global Positioning System). Indirectly, both missions benefited from years of effort to improve the gravity models and the nonconservative force modelling, both major components in the orbit error budget. The situation with GFO is more nuanced. The lack of continuous tracking makes the achievement of radial precision comparable to Jason or T/P more difficult. The challenge then becomes how we are to use the available tracking (SLR, Doppler, altimeter crossovers) to achieve the highest quality orbits. In the absence of the GPS data from GFO, we also had to demonstrate that the SLR data could be used to produce operational orbits with a latency of less than $24 \mathrm{hrs}$. In this paper we describe the quality of the satellite tracking data, the improvements to the force modelling that have been implemented, and the principal factors that affect the GFO radial orbit precision.

\section{Data}

\section{II.A. Satellite Laser Ranging}

The international network of satellite laser ranging stations operates under the aegis of the International Laser Ranging Service (ILRS). ${ }^{6}$ This network is shown in Fig. 2. The network is dominated by stations in the Northern Hemisphere, most notably in Europe. The satellite laser ranging data can have a precision of a few $\mathrm{mm}$, especially for the best stations in the network. In reality, of the 40 or so stations that are in the network, only a subset provide data on a routine basis. In Fig. 3, we show the number of passes of satellite laser ranging data acquired by GFO between January 1, 2005 through March 2006. A satellite pass is defined as a single passage of GFO over a tracking station for which data were acquired. Tracking data are only acquired when the station is staffed, and when the weather permits optical tracking. An additional consideration is the tracking priority assigned to each satellite target by the ILRS. Generally, the satellites lowest in altitude receive the highest priority. The list of tracking priorities is reevaluated on a regular basis, based on scientific needs or changes in the operational status of the user satellites. In this priority scheme, GFO as an altimeter satellite at relatively low altitude $(800 \mathrm{~km})$ receives a high ranking.

From Fig. 3, the Australian station, Yarragadee (YARA), is the prime contributor of SLR data for GFO and supplies $15 \%$ of the data. The remainder of the stations in the NASA network (Monument Peak, California [MNPE], Greenbelt, Maryland [GRF1], and McDonald Observatory, Texas [MCDO1]) supply 11\% of the passes for GFO. The rest of the international network supplies $74 \%$ of the SLR data for GFO, with the largest contributors being Zimmerwald (ZIMM, Bern, Switzerland), Graz (GRAZ, Austria), Herstmonceux (RGO, East Sussex, U.K.), Wettzell (WETT, Germany), and Riyadh (RIYA, Saudi Arabia). The network provides, on average, between 12 to 14 passes of SLR data per day. 


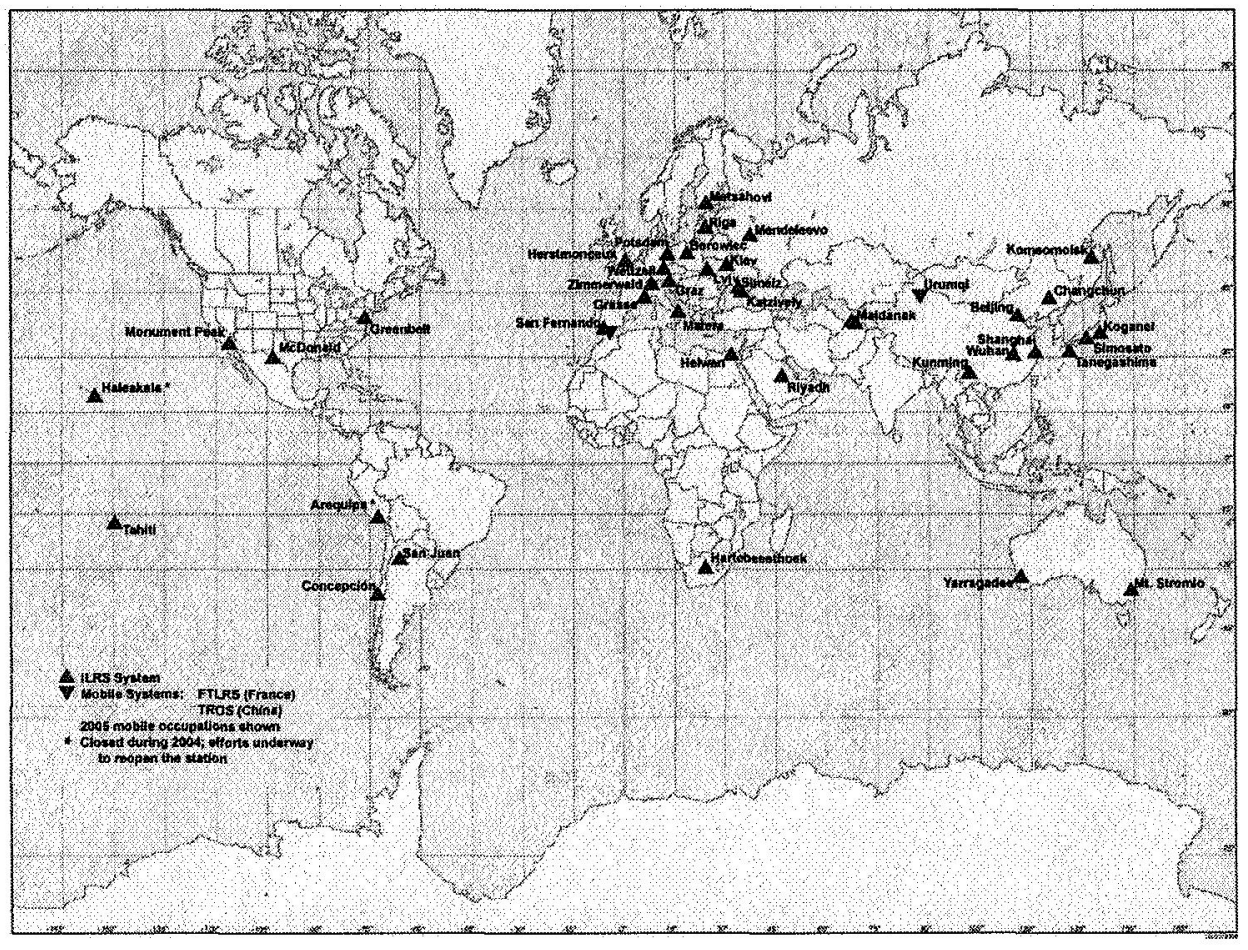

Figure 2. Stations of the International Laser Ranging Service in 2005.

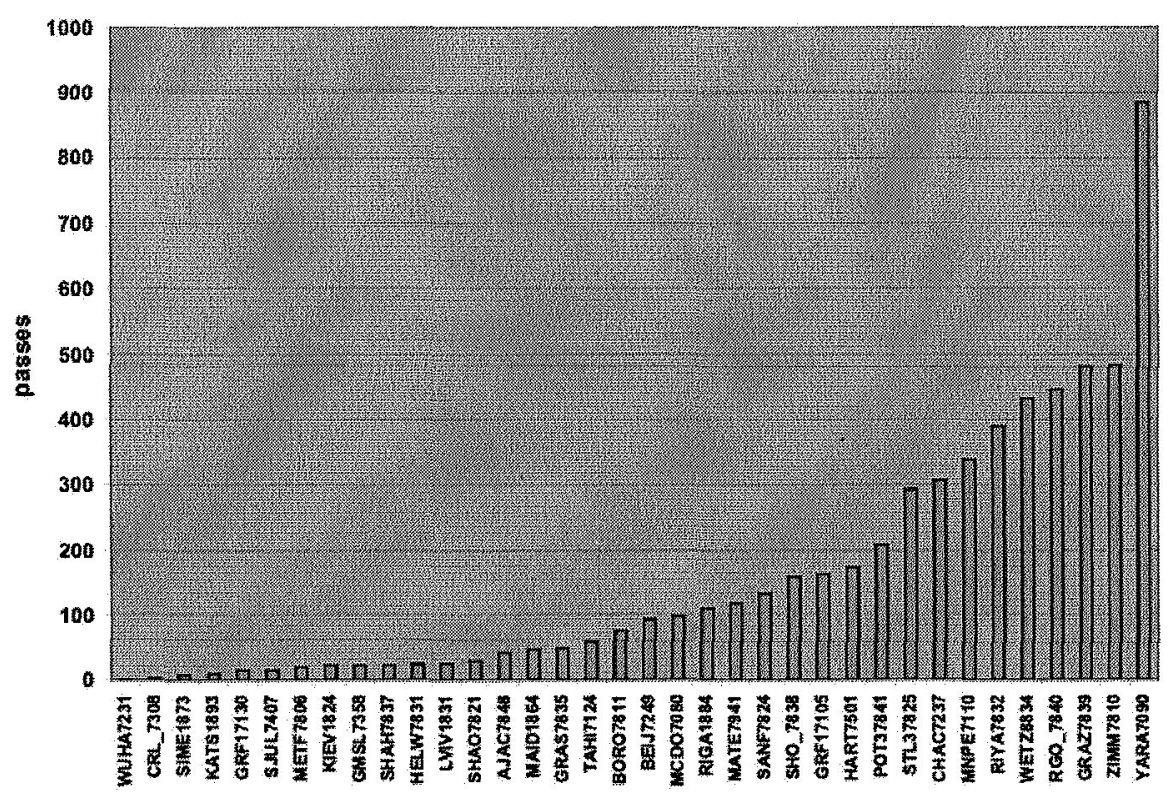

Figure 3. Number of SLR tracking passes of GFO ordered by station, from January 2005 through March 2006 


\section{II.B. Doppler Data}

The Doppler data from GFO are obtained from three stations: Guam, Point Mugu, California, and Prospect Harbor, Maine. The Doppler data are in the style of data from the OPNET stations that tracked GEOSAT, and consist of one-way Doppler data transmitted from GFO, and received at the Earth tracking stations. ${ }^{1,2,7}$ The data have a noise of approximately $2 \mathrm{~cm} / \mathrm{s}$. Although this is quite noisy by modern standards, the Doppler data still help to tie down the orbit when the SLR tracking is sparse. In addition, the Doppler data are needed to obtain new orbit solutions following orbit trim maneuvers.

\section{II.C. Altimeter Data}

In order to supplement the SLR and Doppler tracking, we use the altimeter data supplied by GFO as an additional data type. The data are used in the form of altimeter crossovers. ${ }^{8,9}$ Altimeter crossover data are formed by differencing altimeter ranges from two intersecting passes and interpolating to the point of interesection. ${ }^{10}$ The GFO altimeter crossovers provide dense spatial coverage over the ocean areas. The data are edited in regions with high sea surface variability (greater than $20 \mathrm{~cm}$ ), and in shallow seas (less than $500 \mathrm{~m}$ depth). In addition, a maximum residual edit criterion of $20 \mathrm{~cm}$ is also applied. The altimeter range corrections, as applied in the IGDR (Intermediate Geophysical Data Record), are listed in Table 2. We use a T/P derived tide model, GOT00.2, for the ocean tide altimeter range correction. The GOT00.2 tide model is based on 286 cycles of altimeter data from TOPEX/Poseidon. The GOT00.2 tide model is an update of the GOT99.2 tide model, which was based on 232 cycles of TOPEX/Poseidon altimetry. ${ }^{11}$ The dry troposphere correction is derived from the NOAA Global Forecast System, based on the Global Data Assimilation System. ${ }^{13}$ For the wet troposphere correction we use first the GFO water vapour radiometer (WVR) correction. If the IGDR contains a null field for the GFO WVR, we apply the NCEP model derived value. We tested the IRI2000 ionosphere model, ${ }^{14}$ but did not discern a significant improvement with respect to using the IRI95 $5^{15}$ model for GFO.

Table 2. Altimeter Range Modelling for the GFO IGDR

\begin{tabular}{ll}
\hline Ocean tide & GOT00.2 \\
Earth tide & Cartwright and Eden (updated) ${ }^{12}$ \\
Dry troposphere & NCEP $^{13}$ \\
Wet troposphere & GFO WVR or NCEP $^{13}$ \\
Ionosphere & IRI95 $^{15}$ \\
Inverse barometer & $f(\text { dry troposphere })^{16}$ \\
EM bias & $3.8 \%$ SWH \\
\hline
\end{tabular}

\section{The GFO Orbit Determination System}

\section{III.A. Overview}

The orbit determination system we have designed for GFO imports tracking data and ancillary data from a variety of sources, and delivers three products: the medium precision orbits (MOE's), the precise orbits (POE's), and the ephemeris predictions for the satellite laser ranging stations. The satellite laser ranging data are delivered at least once daily from the tracking stations to the ILRS data centers, at the NASA GSFC Crustal Dynamics Data Information System (CDDIS) in Greenbelt, Maryland and at the Deutsches Geodätisches Forschungsinstitut (DGFI) in Munich, Germany. ${ }^{17,18}$ The data centers exchange their recentlydelivered data daily to ensure that their holdings are equalized so that users can obtain reliably obtain data should one of the data centers be temporarily inaccessible. The GFO Doppler data are delivered electronically from the Naval Space Operations Center (NAVSOC) at Point Mugu, to NASA GSFC Monday through Friday, but not on weekends. The IGDR altimetry data are obtained daily from NOAA. On a typical day, the SLR and Doppler data are imported by early afternoon local time ( 16:00 to 17:00 hrs UT). This means we use SLR and Doppler passes that are obtained through the day of the arc, even up to mid-afternoon UT time. Each MOE arc spans five days ending on the current day, but due to latency of processing at NOAA, altimeter crossovers are included in the MOE's only for the first three days of the MOE arc.

5 of 17 
In addition to the tracking data, we must import other ancillary information from external sources. We obtain daily updates on Earth orientation from the International Earth Rotation Service (IERS) at the Paris Observatory. ${ }^{19}$ Solar flux and geomagnetic data are downloaded daily from NOAA National Geophysical Data Center. ${ }^{20}$ The F10.7 solar flux and geomagnetic indices are used as inputs to atmospheric density models which are required to model atmospheric drag at the GFO altitude.

The MOE orbit is exported daily by $21: 00 \mathrm{hrs}$ to 23:00 hrs UT to data users. The precise orbit (POE) is computed with a latency of three to four weeks. The MOE orbit is computed daily using a sliding window of five days of data. The POE arcs are six days in length and overlap by only one day. The POE's benefit from stabilized values for the Earth orientation, and use altimeter data to form crossovers over the entire data arc. The daily MOE arc is used to create an ephemeris prediction which is supplied to the laser stations to ensure continued tracking for GFO.

Orbit trim maneuvers disrupt normal satellite operations and require special attention. The orbit trim maneuvers maintain the satellite to within \pm $1 \mathrm{~km}$ of the nominal GEOSAT ground track. However they invalidate the predicted ephemerides sent to the laser tracking stations during the previous

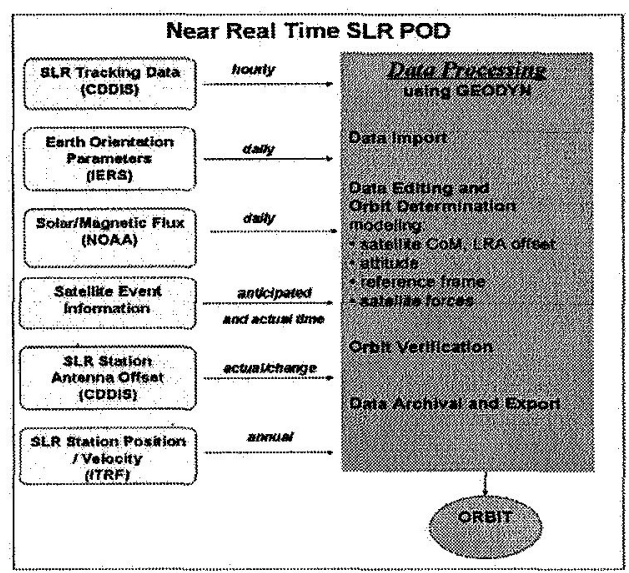

Figure 4. Near-Real-Time Orbit Determination System for GFO. days. In 2005, there were 11 orbit trim maneuvers.

The SLR stations must be notified of the impending maneuver in order to prevent fruitless attempts to track the satellite. NAVSOC supplies post-maneuver state vector predictions for GFO. We propagate this state vector and distribute these predicted ephemerides to the stations to ensure continued tracking of the satellite.

Spacecraft anomalies also perturb normal orbit system operation. If the anomalies cause the spacecraft to deviate from its nominal nadir attitude, the SLR stations must also be notified since the laser retroreflector array might not oriented properly to permit SLR tracking.

We use the NASA GSFC GEODYN orbit determination and orbit parameter estimation program to compute the GFO orbits. This orbit determination program is a batch, least-squares filter that processes numerous types of satellite tracking data. ${ }^{21}$ GEODYN is also used to compute the orbits of $\mathrm{T} / \mathrm{P}$ and Jason$1,{ }^{4}$ and processed the tracking data that was incorporated into the gravity models developed to support GFO. ${ }^{22,23}$ A schematic of the GFO orbit determination system is depicted in Fig. 4 The analysis of the GFO tracking data requires detailed force and measurement modelling which we will summarize in the next sections.

\section{III.B. Force Modelling}

We must model as accurately as possible all the forces that affect the spacecraft trajectory. For altimeter satellites, it is the gravity field induced error, and the nonconservative force model error that are the largest contributors to the radial orbit error budget. ${ }^{3}$

\section{III.B.1. Gravity Field}

We model the gravity field in spherical harmonics using normalized coefficients $\left(\bar{C}_{l m}, \bar{S}_{l m}\right)$ using the equa$\operatorname{tion}^{24}$

$$
U=\frac{G M}{r}+\frac{G M}{r} \sum_{l=2}^{\infty} \sum_{m=0}^{l}\left(\frac{R e}{r}\right)^{l} \bar{P}_{l m}(\sin \phi)\left[\bar{C}_{l m} \cos (m \lambda)+\bar{S}_{l m} \sin (m \lambda)\right]
$$

where $G M$ is the universal constant of gravitation times the mass of the Earth, $l$ is the degree, $m$ is the order, $\bar{P}_{l m}$ are the fully normalized associated Legendre polynomials, $R_{e}$ is the reference radius of the Earth, 
$\phi$ is the latitude, and $\lambda$ is the longitude. By definition, the degree one terms are zero, since we choose the origin of the coordinate system to be at the center of mass of the Earth.

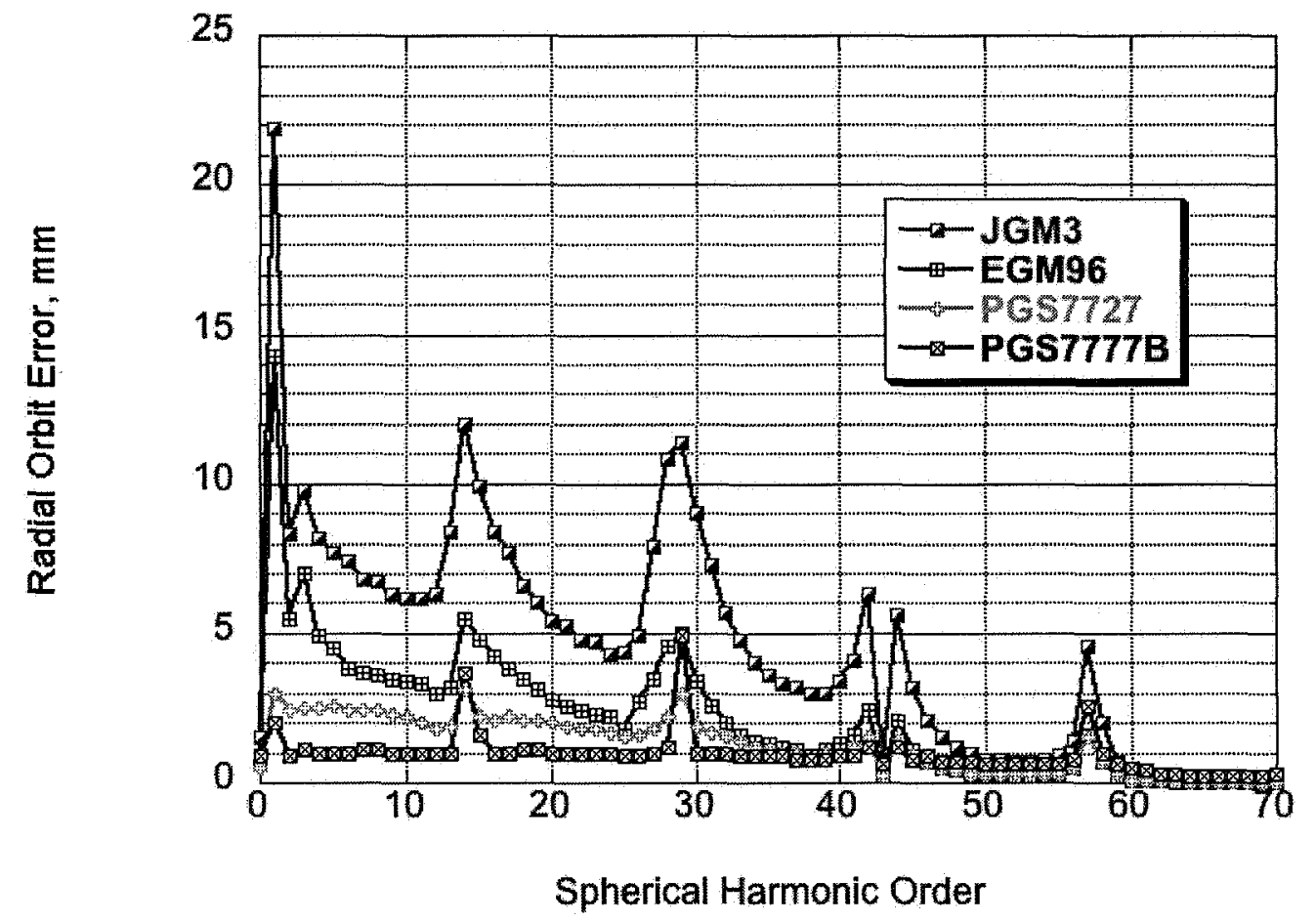

Figure 5. Radial orbit error vs. spherical harmonic order for GFO from the gravity field error covariances through degree and order $\mathbf{7 0}$.

Table 3. Gravity Field Radial Orbit Error for GFO

\begin{tabular}{lc}
\hline Gravity model & $\begin{array}{c}\text { Orbit error } \\
\text { (mm) }\end{array}$ \\
\hline JGM-2 (1993) & 65.2 \\
JGM-3 (1996) & 49.8 \\
EGM96 (1996) & 26.2 \\
PGS7727 (2001) & 13.2 \\
PGS7777b (2003) & 10.0 \\
\hline
\end{tabular}

We currently use the PGS7777b gravity model for GFO orbit production. The model, developed at NASA GSFC, was based on CHAMP and other satellite data, and included data based 54 arcs of GFO tracking data in 2000 to 2001, including SLR, Doppler, and altimeter crossovers. Effectively, this model was tuned for GFO and used as a base the GPS tracking data and precision accelerometry data from CHAMP. ${ }^{23}$ The projected radial orbit error was reduced from $65.2 \mathrm{~mm}$ with JGM-2 ${ }^{25}$ to $49.8 \mathrm{~mm}$ with the JGM3 gravity model, ${ }^{26}$ and $26.2 \mathrm{~mm}$ with EGM96 ${ }^{27}$ to $10 \mathrm{~mm}$ with PGS7777b. The JGM-2 and JGM-3 gravity models were developed for the TOPEX/Poseidon mission, so that although they contained GEOSAT tracking data, they were not specifically tuned to the

GEOSAT orbit. EGM96 included a complete reprocessing of the JGM-2 satellite tracking data, contributions from other satellites, and most importantly much improved altimetry and surface gravity data. EGM96 included GEOSAT Doppler and altimetry data from November 1986 through January 1987 but the the contribution of the GEOSAT data was limited by the short span of data, and the lack of direct ties between the TRANET and OPNET Doppler stations that tracked GEOSAT and the SLR data that in large part defined the low degree field and reference frame for EGM96.

The PGS7727 model, developed at GSFC as a derivative of EGM96, included both TOPEX/GFO (dualsatellite) and GFO/GFO (single satellite) altimeter crossovers, including the SLR data to GFO. The addition of these data reduced the radial orbit error to $13.2 \mathrm{~mm} .{ }^{22}$ We show the gravity field error as a function of spherical harmonic order in Fig. 5. The JGM-3 and EGM96 gravity models had large errors at order one, and at the resonant and the near-resonant orders (e.g., $m=14,15,28,29$ ). GFO has heightened sensitivity to the gravity field at order one due to the m-daily perturbations. ${ }^{24} \mathrm{It}$ is clear that the GEOSAT Doppler tracking alone in these gravity solutions was insufficient to model adequately these gravity field terms for GFO. The 
addition of GFO SLR and altimeter crossover data in PGS7727 and PGS7777b was highly effective in tuning the gravity field at those orders.

The PGS7777b includes secular variations in $\bar{C}_{20}, \bar{C}_{21}$, and $\bar{S}_{21}$, and annual variations $\delta \bar{C}_{20}, \delta \bar{C}_{30}$ and $\delta \bar{C}_{40}$ (see Table 4). The periodic variations in the low degree zonals are derived from an independent analysis of SLR and DORIS tracking data spanning two decades. ${ }^{28}$

\begin{tabular}{lc}
$\begin{array}{l}\text { Table 4. } \\
\text { Terms }\end{array}$ & PGS7777b \\
\hline Term & Value-Variable Gravity \\
\hline $\bar{C}_{20}$ (Secular) & $1.258 \times 10^{-11} / \mathrm{yr}$ \\
$\bar{C}_{21}$ (Secular) & $-0.337 \times 10^{-11} / \mathrm{yr}$ \\
$\bar{S}_{21}$ (Secular) & $1.606 \times 10^{-11} / \mathrm{yr}$ \\
$\delta C_{20}$ Annual Cosine & $3.4163 \times 10^{-11}$ \\
$\delta C_{20}$ Annual Sine & $10.2115 \times 10^{-11}$ \\
$\delta C_{30}$ Annual Cosine & $-0.3148 \times 10^{-11}$ \\
$\delta C_{30}$ Annual Sine & $0.1877 \times 10^{-11}$ \\
$\delta C_{40}$ Annual Cosine & $-3.6574 \times 10^{-11}$ \\
$\delta C_{40}$ Annual Sine & $-4.1864 \times 10^{-11}$ \\
\hline
\end{tabular}

* The epoch of the PGS7777b solution is 1998.0.

\section{III.B.2. Macromodel}

The solar radiation pressure, planetary radiation pressure (due to the Earth's albedo and thermal emission), and the atmospheric drag are modelled using a macromodel. ${ }^{29}$ Analagous to TOPEX, the GFO spacecraft is modelled as a series of elemental flat plates, and the contributions of each plate are vectorially summed to obtain the total acceleration (See Fig. 6).

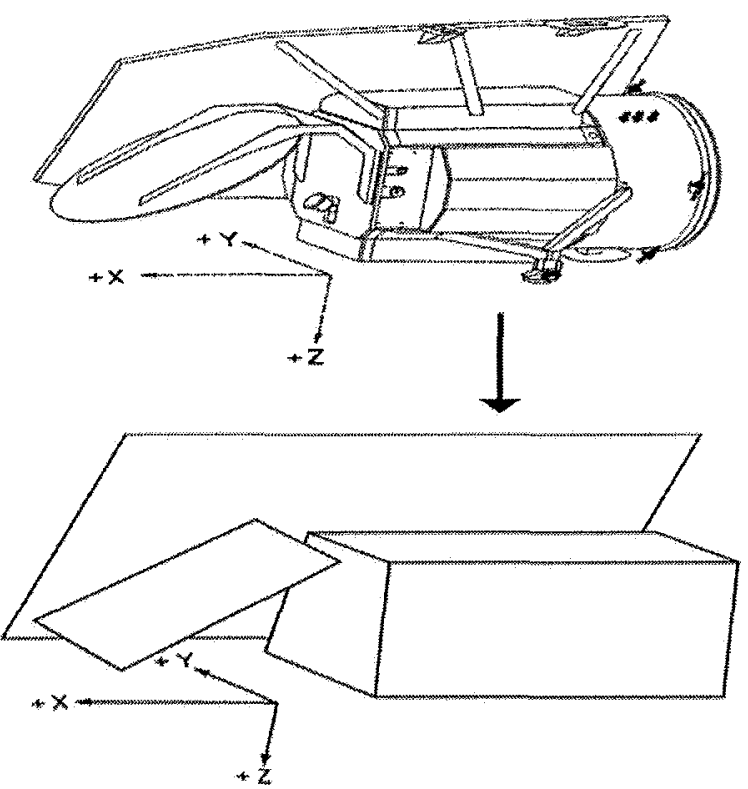

Figure 6. Schematic of macromodel for GFO.

We model GFO using 8 elemental plates representing the $+X,-X,-Y,+Z,-Z$ spacecraft faces, the front 
side of the solar array, and front and the back of the radar altimeter. Self-shadowing complicates the development of the macromodel. The $+\mathrm{Y}$ face of the spacecraft is obscured by the solar array and does not enter into the computations. Since the solar array tracks the Sun, the $-\mathrm{Z}$ surface are shadowed. Hence, in the macromodel, the $-Z$ reflectivity parameters are set to zero. Thus, the $-Z$ plate participates in the drag calculations, but not in the radiation pressure calculations. We list the macromodel areas, and the specular $(\beta)$ and diffuse $(\delta)$ reflectivities in Table 5 which are used in the current generation of MOE and POE orbits. The GFO macromodel does not account perfectly for self-shadowing effects that might vary with beta prime (the angle of the Sun above or below the orbit plane). In addition, the macromodel does not include radiation interactions between surfaces. Also, unlike $\mathrm{T} / \mathrm{P}$, a detailed thermal model for POD was not developed. Notwithstanding these imperfections, the GFO macromodel is an improvement over using a simple cannonball for the nonconservative force modelling.

Table 5. GFO Macromodel Parameters

\begin{tabular}{lcc}
\hline Plate & $\begin{array}{c}\text { Area } \\
\left(\mathrm{m}^{2}\right)\end{array}$ & $\begin{array}{c}\text { Reflectivities } \\
(\beta, \delta)\end{array}$ \\
\hline +X face & 0.320 & $(0.36,0.09)$ \\
-X face & 0.736 & $(0.44,0.11)$ \\
-Y face & 2.370 & $(0.526,0.132)$ \\
+Z face & 2.450 & $(0.555,0.139)$ \\
-Z face & 0.750 & $(0.0,0.0)^{*}$ \\
Solar array (front) & 3.987 & $(0.144,0.04)$ \\
Radar altimeter (front) & 0.880 & $(0.688,0.172)$ \\
Radar altimeter (back) & 0.880 & $(0.688,0.172)$ \\
\hline
\end{tabular}

* The $-Z$ plate surface area is reduced to account for shadowing by the solar array. In addition, this plate participates in the macromodel drag calculations, but not the radiation pressure calculations.

\section{III.B.3. Other Force model effects}

The solid earth tides are modelled with $k_{2}=0.300$ and $k_{3}=0.093$, and special modelling for the free core nutation. ${ }^{27}$ The ocean tides use as a background the GOT99.2 ocean tide model, ${ }^{11}$ derived from TOPEX/Poseidon altimetry. The PGS7777b gravity solution also included adjustments for the resonant tide terms (as described in Ref. 27) and these are overlain on the background tide model. We use the MSIS86 atmospheric density model. ${ }^{30}$ The planetary radiation pressure due to the Earth's shortwave and longwave flux is modelled as described in Ref. 31, where the total acceleration at each time step is computed as a double summation over both the contribution from each plate and the elements on the surface of the Earth in view of the satellite. The third body perturbations due to the Sun, Moon, and planets are modelled using the DE403 set of planetary ephemerides. ${ }^{32}$

\section{III.C. Measurement Modelling}

\section{III.C.1. Station coordinates}

The SLR station coordinates are based on the ITRF2000 solution. ${ }^{33}$ Coordinates for new SLR stations that were not originally part of the ITRF2000 solution (for example the new station at Mt Stromlo, Australia, rebuilt after the destruction of the old station there due to bushfires in January 2003) were obtained from ground survey ties, or independent adjustments using data to the satellites Lageos-1 and Lageos-2. The TOPEX POD team applied a number of corrections to the coordinates of some of the SLR stations in ITRF2000. The SLR station coordinates used for GFO are identical to those used in the production of the precise orbits for the TOPEX/Poseidon geophysical data records. NAVSOC supplied a priori coordinates for the Doppler stations which were adjusted to the SLR frame. Ocean loading corrections are computed using the GOT00.2 ocean tide model and include the M2, S2, K2, K1, O1, P1, Q1, Mf, Mm, and Ssa tidal 
constituents. We include both the vertical, as well as the eastward and northward displacements due to ocean loading. The ocean loading correction diminishes as the distance of the station from the coast increases. The ocean loading corrections must be included in order to exploit the precise SLR data. For example, for the M2 constituent, the vertical displacement load tide might be $10 \mathrm{~mm}$ or less for the Mt. Stromlo SLR station, but 20 to $30 \mathrm{~mm}$ for the San Fernando SLR station.

\section{III.C.2. Measurement Offset Modelling}

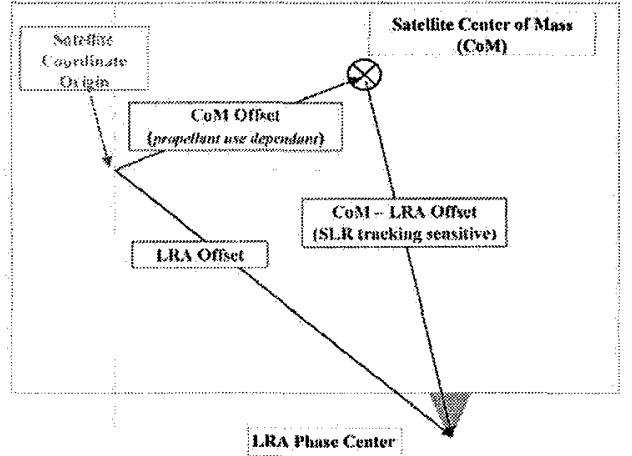

Figure 7. GFO laser retroreflector array geometry in the spacecraft coordinate system.

It is necessary to define two vectors in the spacecraft coordinate system: (1) the location of the spacecraft center-of-mass (CoM), and (2) the location of the phase center of the laser retroreflector array. While the LRA is fixed, the CoM varies with propellant usage (see Fig. 7). Early in the mission, the initial analyses of SLR data showed a large mean in the residuals. The SLR data were used to estimate the LRA offset. We cannot say whether the adjustment was a results of an error in the LRA location, or an error in the specification of the CoM in the spacecraft coordinate system. Nonetheless, the application of the new offset reduced the SLR residual mean from $-2.5 \mathrm{~cm}$ to zero, over the test period. The offset adjusted by $-6.3 \mathrm{~cm}$ in $X,-1.1 \mathrm{~cm}$ in $Y$, and $+11.1 \mathrm{~cm}$ in $\mathrm{Z}$.

\section{III.C.8. Attitude Modelling}

The GFO spacecraft follows a yaw steering algorithm that maintains the solar array pointed at the

Sun, while the radar altimeter is pointed at nadir. Unlike TOPEX/Poseidon, where quaternions are routinely available during off-nominal attitude regimes, for GFO we must rely completely on an analytical description of the spacecraft attitude. We obtained a limited amount of quaternion data for GFO early in the mission and compared the orientation angles for GFO computed from the analytical attitude model with those from the satellite telemetry. As depicted in Fig. 8, the differences are on average less than $0.3^{\circ}$ in roll, pitch, and yaw. Thus, as long as GFO follows its prescribed attitude law, we will be able to orient the macromodel correctly in inertial space. In addition, assuming a maximum $0.3^{\circ}$ error in attitude, we are assured that the error in the LRA measurement offset correction due to incorrect attitude knowledge will be less than 4 mm.

\section{Results}

\section{IV.A. MOE orbits}

For the MOE orbits, we adjust the spacecraft state, a drag coefficient $\left(c_{d}\right)$ per day, and empirical onceper-revolution (opr) accelerations along-track and cross-track to the orbit once per arc. Range-rate and troposphere biases are adjusted for the Doppler data. Pass-by-pass biases are adjusted for some of the non-core SLR stations of the ILRS. The Doppler data are weighted at $2 \mathrm{~cm} / \mathrm{s}$, whereas the SLR data are weighted at $10 \mathrm{~cm}$. However, the data from some SLR stations are downweighted to between $60 \mathrm{~cm}$ and 1 meter. The altimeter crossover data are weighted at $10 \mathrm{~cm}$. All arcs with altimeter crossover data adjust an altimeter timing bias whose typical value is between 0.8 to 1.3 msecs. The adjustment of a timing bias on the altimeter data makes the time tag on the altimetry consistent with the time recorded at the SLR stations.

Prior to January 20, 2004, the PGS7727 gravity model was used for MOE production. The PGS7777b gravity model has been used since January 20, 2004. The altimeter crossovers were introduced into the solutions for the MOE orbits starting on February 4, 2004. The crossovers were introduced to strengthen the solution for the MOE orbits, and compensate for diminished tracking schedules at the NASA SLR stations due to budget issues. 


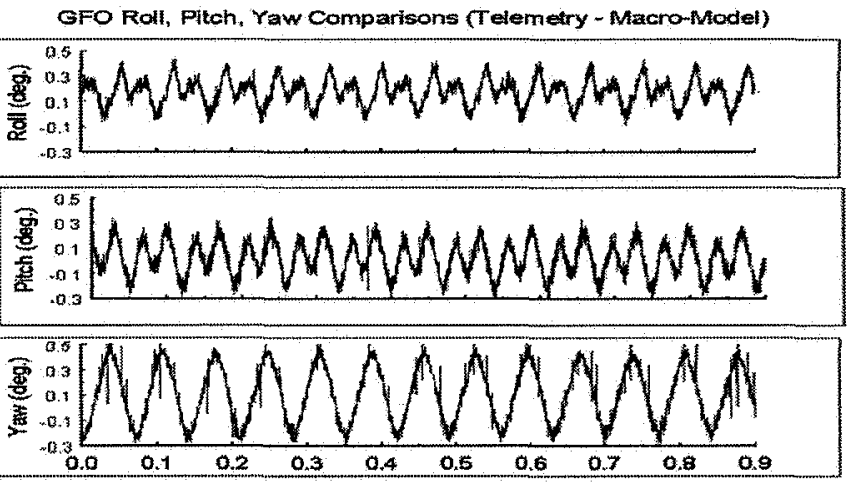

Days From Arc Start (900811)

Figure 8. Comparison of GFO roll, pitch, and yaw from telemetry and from analytical model.

We show the RMS of fit for the daily MOE arcs in Fig 9. The long-term average daily RMS of fit is 6.1 $\mathrm{cm}$ for the SLR data, and $7.3 \mathrm{~cm}$ for the crossover data. However, there are large variations, and there is a correlation with the F10.7 solar flux with the RMS of fit degrading during high flux periods. The RMS of fit is also typically higher for arcs that start after maneuvers. The GFO orbit maneuvers almost always cause a disruption in the SLR tracking at some level, even if the predicted post-maneuver ephemerides are distributed in a timely fashion. In addition, for reasons of latency, for the first 2-3 days, the daily postmaneuver arcs do not contain any altimeter crossover data. Thus, the MOE arcs in the immediate aftermath of maneuvers have less dynamical strength than the 'normal' five-day MOE arcs.

Spacecraft anomalies will also affect the quality of the MOE orbits. Sometimes the cause of the degraded MOE orbit quality is not always obvious. For example, from November 4 to November 13, 2004, the RMS of fit to the SLR data in the daily arcs increased to between 13 and $25 \mathrm{~cm}$. The precise orbits also show an increase in the SLR RMS of fit at that time, though only to $9 \mathrm{~cm}$ and only for the six-day arc beginning on November 7,2004 . It is likely there was a satellite or a data glitch on approximately November 10 or 11,2004 . Because of the sliding window used for the MOE's, if a satellite or a data anomaly is not properly identified, then the effects can be felt in the MOE's for several days.

Another way to assess orbit consistency is through the use of orbit overlap comparisons. We routinely compare the orbits from sequential MOE arcs, as long as there is no interruption due to a maneuver. Hence, the orbit overlaps usually contain four days of common data. We must be cautious in interpreting these statistics as they will be optimistic in their orbit quality assessment since they have so much data in common. We show these daily overlaps in Fig. 10 and summarize the full statistics in Table 6. The mean RMS overlaps are $5.11 \mathrm{~cm}$ radially, $11.89 \mathrm{~cm}$ cross-track, and $23.82 \mathrm{~cm}$ along-track. We omit the first arcs after maneuvers or spacecraft anomalies which would otherwise distort the results. We see once again, that maneuvers always cause a disruption in orbit consistency, and that it takes several days for the orbit statistics to stabilize. A semiannual signal is evident in the radial and cross-track orbit overlaps which might be related to errors in modelling of the atmospheric drag or the radiation pressure.

\section{IV.B. POE Results}

The POE orbits are computed with a latency of three to four weeks. The extra latency allows extra SLR and altimeter data to be imported to cover more of the data arc. In addition, the POE processing can take advantage of any independent analysis of the SLR or altimeter data that points to station, data, or spacecraft anomalies that were not diagnosed for the MOE production. Finally, the latency permits us to 


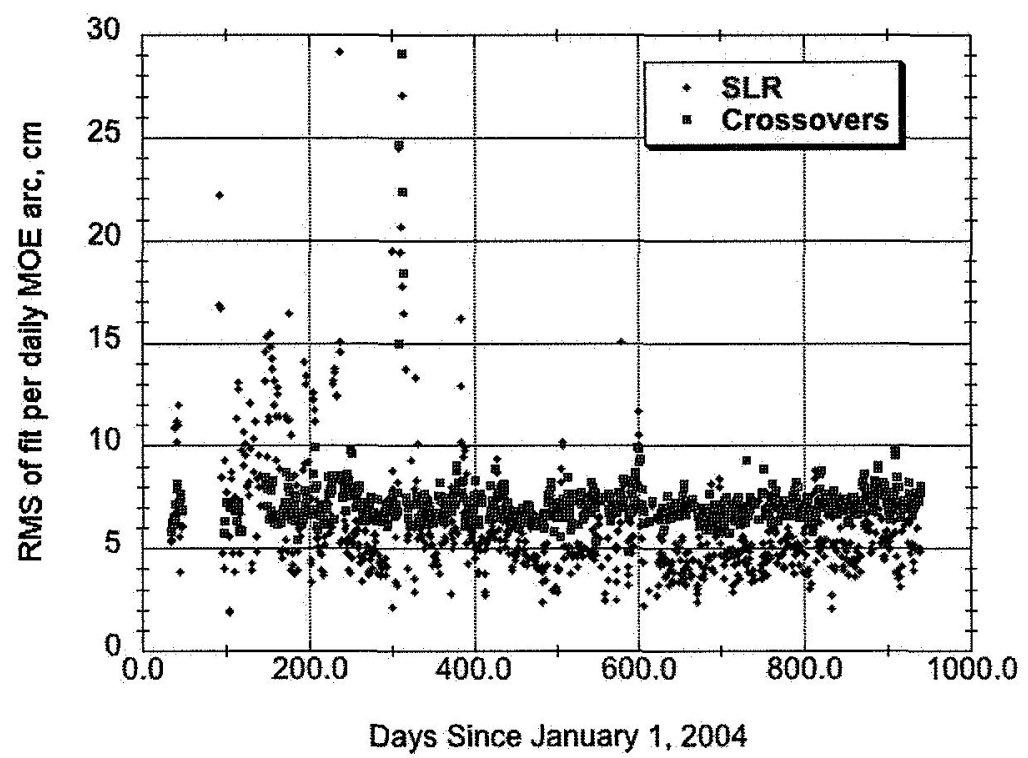

Figure 9. SLR and crossover RMS of fit for daily MOE (medium precision orbit) arcs from February 4, 2004 through July 28, 2006 .

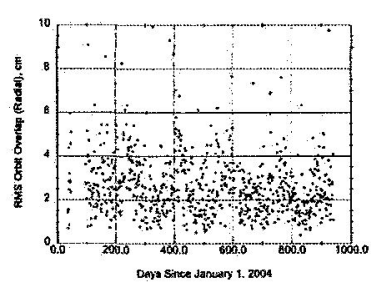

(a) Radial

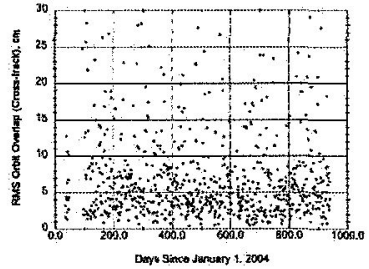

(b) Cross-track

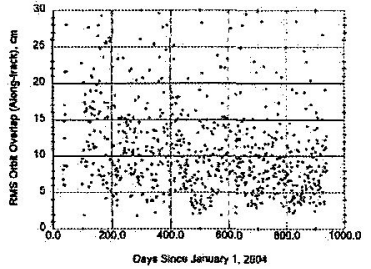

(c) Along-track

Figure 10. RMS orbit overlaps for daily sequential MOE orbits, radial, along-track and cross-track to the orbit. The orbit overlaps are usually 4 days in length, except after orbit trim maneuvers. 
Table 6. Orbit Overlap Statistics for Daily MOE arcs from February 4, 2004 to July 28, 2006

\begin{tabular}{lcccc} 
& \multicolumn{4}{c}{ MOE Orbit Statistics $(\mathrm{cm})^{*}$} \\
& Mean & Median & Minimum & Maximum \\
\hline Radial & 5.11 & 2.49 & 0.38 & 479 \\
Cross-track & 11.89 & 42.39 & 0.43 & 730 \\
Along-track & 23.82 & 97.62 & 1.78 & 1521.6 \\
\hline
\end{tabular}

* The orbit statistics are computed over 836 daily MOE arcs. Arcs on the first day after a maneuver or a spacecraft anomaly are omitted from this summary.

import stabilized and updated values for the Earth orientation and the solar flux. The set of estimable parameters is the same as for the MOE arcs, except for the empirical accelerations and drag coefficients $\left(c_{d}\right.$ 's).

Since altimeter data are available for the full six days of the data arc, more crossovers can be computed, especially since the number of potential crossovers is proportional to $n^{2}$, where $n$ is the number of orbital revolutions in a data arc. A typical POE arc has on average 2600 crossovers, whereas a typical MOE arc has only 675 crossovers over the first three days of the five-day arc. The extra data mean that a denser parameterization can be employed to accommodate force model error. Hence, for the POE's we apply the same parameterization as for the TOPEX POE's: we adjust opr's along-track and cross-track once per day, and we adjust drag coefficients $\left(c_{d}\right.$ 's) every eight hours. Between January 4, 2000 and June 28, 2006, we computed and exported 423 POE arcs. At first, we computed the GFO POE's with the PGS7727 gravity model, however once the PGS7777b gravity model became available in early 2004, all the earlier POE arcs were recomputed with the newer gravity model. For the PGS7777b generation POE's, the average SLR RMS of fit is $4.37 \mathrm{~cm}$ and the average crossover RMS of fit is $7.51 \mathrm{~cm}$. We show the RMS of fit in Fig. 11 and summarize the RMS by year in Table 7. The RMS for all data types (SLR, crossovers, Doppler) peaks in 2001 and 2002. The F10.7 radio flux from the Sun peaked between January 2000 and early 2002 , so these peaks in the GFO RMS of fit are roughly coincident with the peak of solar cycle 23. The RMS of fit of a GFO POE arc may vary with the solar cycle for two reasons: (1) At the peak of the solar cycle, GFO is more susceptible to mismodelling from atmospheric drag; (2) For a single-frequency altimeter, the ionosphere error is more substantial near the solar cycle peak, than near the solar minimum.

We also examine the orbit overlap statistics for the POE arcs (see Table 8), and compare these to the MOE orbit overlap statistics (see Table 6. From these statistics, we can guess that the radial orbit precision is 2.5 times the mean radial orbit overlap of $1.84 \mathrm{~cm}$.

In order to make an orbit accuracy assessment for GFO, we can estimate the sea surface variability using the GFO orbits, and then repeat the calculation after adjusting the GFO orbits using TOPEX/GFO crossovers. We illustrate this process in Fig. 12. We first computed the GFO sea-surface variability using the PGS7727 orbits and then adjusted the GFO orbits empirically relative to TOPEX/Poseidon. The resultant sea surface variability is $8.3 \mathrm{~cm}$, compared to $9.5 \mathrm{~cm}$ before adjustment. The RSS difference between the sea surface variability maps is $4.6 \mathrm{~cm}$, and can be taken as an estimate of the radial orbit error for GFO (relative to TOPEX). This analysis was done using the PGS7727 orbits early in the mission, so we have confidence that the GFO POE's now computed with the PGS7777b gravity model have a radial precision of $5 \mathrm{~cm}$.

\section{Conclusion}

The GFO mission was rescued by the on-board presence of the laser retroreflector, and the demonstration of near-real time POD using a combination satellite laser ranging data, Doppler and altimeter crossover data. Starting in January 2000, we have produced a 6.5 year span of POE orbits for the GFO GDR. With these orbits, altimeter analysis has shown that GFO is a Poseidon-class altimeter, and provides an important supplement to Jason-1, TOPEX/Poseidon (T/P), ERS and ENVISAT. GFO altimeter data are being used to monitor inland lakes, and has been used for near-real-time monitoring of hurricanes and to detect the 2004 Boxing Day tsunami in the Indian Ocean. 


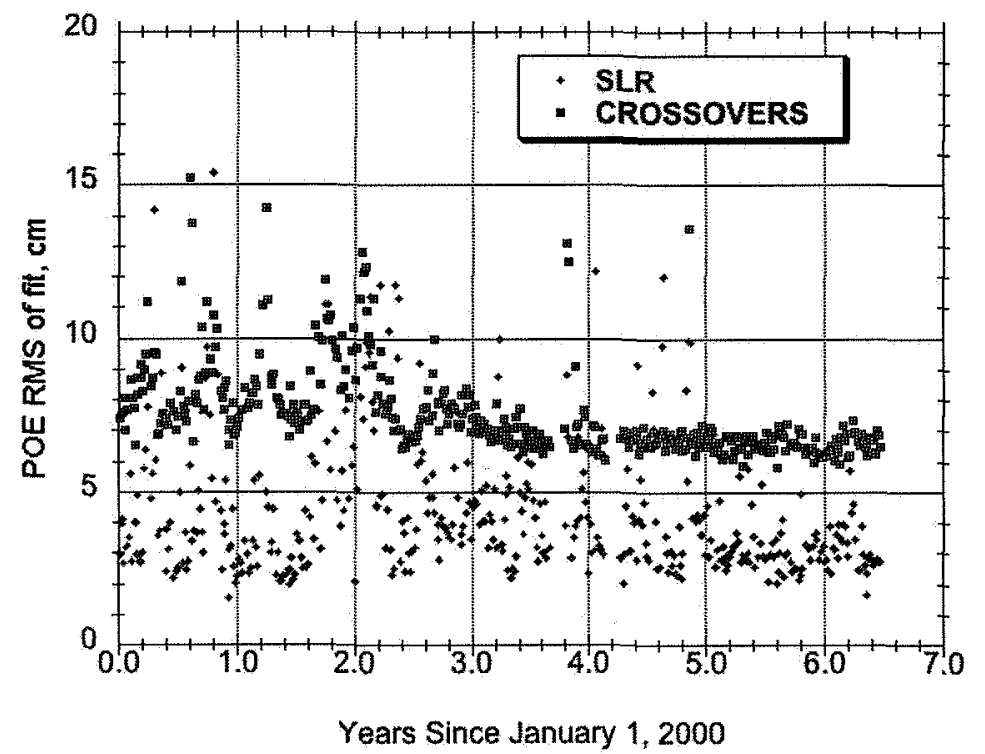

Figure 11. SLR and Crossover RMS for GFO POE arcs from January 4, 2000 to June 28, 2006.

Table 7. SLR, Crossover, and Doppler RMS of fit for GFO POE Arcs

\begin{tabular}{ccccc}
\hline & & \multicolumn{3}{c}{ Average RMS of Fit } \\
Year & $\begin{array}{c}\text { Number } \\
\text { of Arcs }\end{array}$ & $\begin{array}{c}\text { SLR } \\
(\mathrm{cm})\end{array}$ & $\begin{array}{c}\text { Crossovers } \\
(\mathrm{cm})\end{array}$ & $\begin{array}{c}\text { Doppler } \\
(\mathrm{cm} / \mathrm{s})\end{array}$ \\
\hline 2000 & 67 & 4.68 & 8.41 & 1.74 \\
2001 & 60 & 4.70 & 8.64 & 1.93 \\
2002 & 66 & 5.39 & 8.12 & 2.10 \\
2003 & 63 & 4.45 & 7.12 & 1.93 \\
2004 & 62 & 4.49 & 6.80 & 1.75 \\
2005 & 71 & 3.26 & 6.57 & 1.90 \\
2006 & 34 & 3.18 & 6.58 & 1.54 \\
\hline All & 423 & 4.37 & 7.51 & 1.89 \\
\hline
\end{tabular}

Table 8. Orbit Overlap Statistics for POE arcs from January 4, 2000 to June 28,2006

\begin{tabular}{lcccc}
\hline & \multicolumn{4}{c}{ POE Orbit Statistics $(\mathrm{cm})^{*}$} \\
& Mean & Median & Minimum & Maximum \\
\hline Radial & 1.84 & 1.39 & 0.14 & 15.39 \\
Cross-track & 11.56 & 6.15 & 0.57 & 569.56 \\
Along-track & 13.50 & 8.48 & 0.83 & 136.82 \\
\hline
\end{tabular}

* The orbit statistics are computed over 316 overlapping POE arcs. 

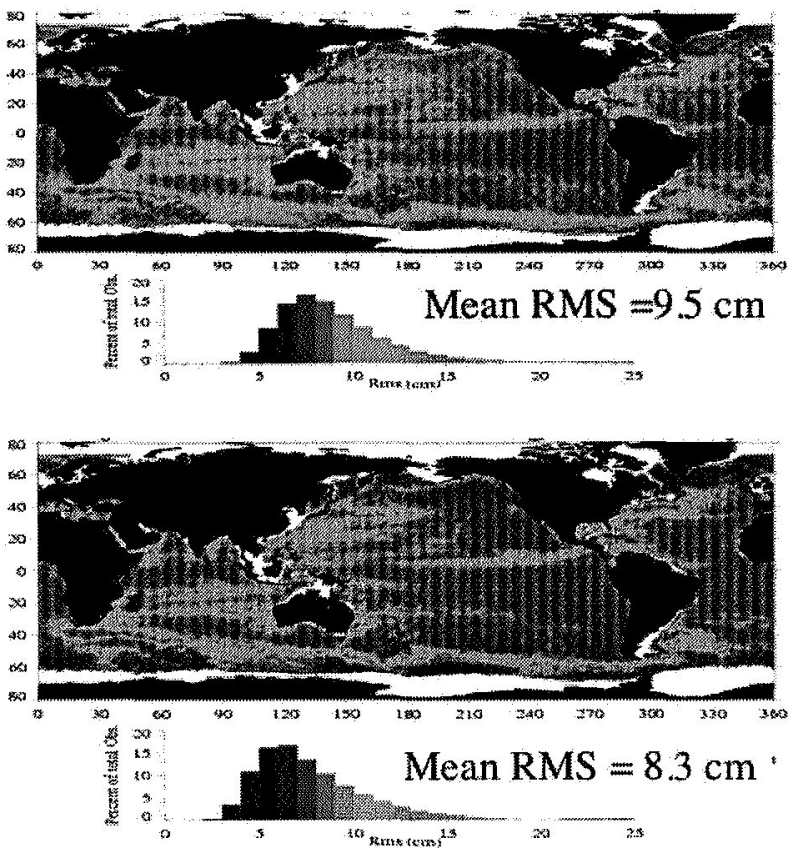

Figure 12. GFO orbit error relative to TOPEX/Poseidon inferred from the mean RMS of the GFO sea surface variability: Top figure, before empirical correction; Bottom figure after empirical correction using TOPEX

The precision orbit lies at the heart of the altimeter measurement, providing the reference frame from which the radar altimeter measurements are made. The NASA GSFC Planetary Geodesy Laboratory produces a daily GFO orbit with a latency of less than $24 \mathrm{hrs}$ and a precise orbit with a latency of three to four weeks. The modelling for the MOE and the POE orbits incorporates improvements to the gravity field, the nonconservative force model, and to the measurement model. After many improvements, the expected radial accuracy is approximately $5 \mathrm{~cm}$ for the POE, and between 10 to $20 \mathrm{~cm}$ for the daily MOE. Further improvements are possible for the MOE and POE orbits, for example using the GRACE generation gravity models, improved ionosphere corrections from the GPS Ionosphere Models, and more sophisticated handling of the nonconservative forces.

An important lesson from GFO is that altimeter missions should always carry multiple means of tracking. Not only can they serve as a backup if one system fails, but the multiplicity of data types allows us to directly intercompare orbits computed using different data and verify the orbit quality, as we have done so successfully on TOPEX/Poseidon and on Jason-1., 


\title{
Acknowledgments
}

We are grateful to the ILRS and to the personnel at the many SLR tracking stations for their continued support of GFO. Carey Noll (NASA GSFC) provided the ILRS network map (Figure 2). We acknowledge Despina Pavlis (SGT Inc.) and John McCarthy (SGT Inc.) for their work in maintaining the GEODYN and SOLVE programs, and without whose efforts the precise orbits for GFO could not have been computed. David Rowlands (NASA GSFC) verified the implemenation of the altimeter crossover model for GFO in GEODYN. Scott Luthcke (NASA GSFC) performed the GFO quaternion comparison illustrated in Figure 8. Yoaz Bar-Sever (JPL) developed the analytical attitude model for GFO.

This work was supported by the NASA Physical Oceanography Program and the National Oceanic and Atmospheric Administration through the Laboratory for Satellite Altimetry.

\section{References}

\begin{abstract}
${ }^{1}$ Haines, B.J., Born, G.H., Rosborough, G.W., Marsh, J.G., and Williamson, R.G., "Precise Orbit Computation for the GEOSAT Exact Repeat Mission," J. Geophys. Res.-Oceans, 95(C3), March 15, 1990.

${ }^{2}$ Haines, B.J., Born, G.H., Williamson, R.G. and Koblinsky C.J., "Application of the GEM-T2 Gravity Field to Altimetric Satellite Orbit Computation," J. Geophys. Res.-Oceans, 99(C8), Aug. 15, 1994.

${ }^{3}$ Marshall, J.A., Zelensky, N.P., Klosko, S.M., Chinn, D.S., Luthcke, S.B., Rachlin, K.E., and Williamson, R.G., "The Temporal and Spatial Characteristics of TOPEX/Poseidon Radial Orbit Error," J. Geophys. Res., Oceans, 100(C12), Dec. 15, 1995, pp. 25331-25352.

${ }^{4}$ Luthcke, S.B., Zelensky, N.P., Rowlands, D.D., Lemoine, F.G., and Williams, T.A., "The 1-cm Orbit: Jason-1 Precision Orbit Determination Using GPS, SLR, DORIS, and Altimeter data," Marine Geodesy, 26(3-4), July-Dec., 2003, pp. 261-284.

${ }^{5}$ Haines, B.J., Bar-Sever, Y., Bertiger, W.I., Desai, S., and Willis, P., "One-cm Orbit Determination for Jason-1: New GPS-Based Strategies," Marine Geodesy, 27(1-2), Jan.-June, 2004, pp. 299-318.

${ }^{6}$ Pearlman, M.R., Degnan, J.J., and Bosworth, J.M, "The International Laser Ranging Service," Advances in Space Research, 30(2), July 2002, pp. 135-143.

${ }^{7}$ Anderle, R.J., "Doppler Satellite Measurements and Their Interpretation," Space Geodesy and Geodynamics, edited by A. J. Anderson and A. Cazenave, Academic Press, New York, 1986, pp. 113-168.

${ }^{8}$ Shum, C.K., Yuan, D.N., Ries, J.C., Smith, J.C., Schutz, B.E., and Tapley, B.D., "Precision Orbit Determination for the GEOSAT Exact Repeat Mission, J. Geophys. Research-Oceans, 95(C3), March 15, 1990.

${ }^{9}$ Scharroo, R. and Visser, P., "Precise Orbit Determination and Gravity Field Improvement for the ERS Satellites," $J$. Geophys. Res.-Oceans, 103(C4), April 15, 1998, pp. 8113-8127.

${ }^{10}$ Rowlands, D.D, Pavlis, D.E., Lemoine, F.G., Neumann, G.A., and Luthcke, S.B., "The Use of Laser Altimetry in the Orbit and Attitude Determination of Mars Global Surveyor," Geophys. Res. Lett., 26(9), 1999, pp. 1191-1194.

${ }^{11}$ Ray, R.D., "A Global Ocean Tide Model from TOPEX/Poseidon altimetry: GOT99.2," NASA TM-1999-209478, NASA Goddard Space Flight Center, September 1999.

${ }^{12}$ Cartwright, D.E., and Eden A.C., "Corrected Tables of Tidal Harmonics," Geophys. J. R. Astr. Soc., 17(5), 1973, pp.
\end{abstract} 619-622.

${ }^{13}$ National Centers for Environmental Prediction (NCEP), Global Forecast System, http://www.emc.ncep.noaa.gov/modelinfo/index.html.

${ }^{14}$ Bilitza, D., "International Reference Ionosphere 2000," Radio Science, 36(2), March/April 2001, pp. 261-275.

${ }^{15}$ Bilitza, D., International Reference Ionosphere - Status 1995/96," Adv. Space Res., 20(9), 1997, pp. 1751-1754.

${ }^{16}$ Wunsch C., and D. Stammer, "Atmospheric loading and the oceanic 'inverted' barometer effect," Rev. Geophys., 35, 1997, pp. 79-107.

${ }^{17}$ Crustal Dynamics Data Information System, URL: http://cddis.gsfc.nasa.gov/cddis.html.

${ }^{18}$ Deutsches Geodätisches Forschungsinstitut, ILRS Global-/EUROLAS Data Center (EDC), URL: http://dgfi2.dgfi.badwmuenchen.de/index.php?id $=131 \& \mathrm{~L}=0$.

${ }^{19}$ International Earth Rotation and Reference Systems Service, URL: http://hpiers.obspm.fr/

${ }^{20}$ National Geophysical data Center (NGDC): NOAA Satellite and Information Service, Solar Data Services: Data pertaining to solar activity and the upper atmosphere, URL: http://www.ngdc.noaa.gov/stp/SOLAR/solar.html

${ }^{21}$ Pavlis, D.E., Poulose, S., and J.J. McCarthy, GEODYN Operations manuals, contractor report, SGT Inc., Greenbelt, Maryland, 2006.

${ }^{22}$ Lemoine, F., Rowlands, D., Luthcke, S., Zelensky, N., Chinn. D., Pavlis, D., and Marr, G., "Precise Orbit Determination for the GEOSAT Follow-On Using Satellite Laser Ranging Data and Intermission Altimeter Crossovers," Proceedings of the 2001 Flight Mechanics Symposium, NASA/CP-2001-209986, edited by J. P. Lynch, June 2001, pp. 377-392.

${ }^{23}$ Lemoine, F.G., Cox, C.M, Zelensky, N.P, Luthcke, S.B., Thompson, B.F, Rowlands, D.D, and Chinn, D.S., and Nerem, R.S, "Gravity models from CHAMP and other satellite data," Eos Trans. AGU, 84(46), Fall Meeting Suppl., Abstract G32A$0730,2003$.

${ }^{24}$ Kaula, W.M., Theory of Satellite Geodesy: Applications of Satellites to Geodesy, Dover Publications, Inc., Mineola, N.Y., 2000.

${ }^{25}$ Nerem, R.S, and 20 coauthors, "Gravity Model Development for TOPEX/Poseidon: Joint Gravity Model-1 and Model-2," J. Geophys. Res.-Oceans, 99(C12), 1994, pp. 24421-24447. 
${ }^{26}$ Tapley, B.D., and 14 coauthors, "The Joint Gravity Model-3," J. Geophys. Res.-Solid Earth, 101(B12), 1996, pp. 2802928049.

${ }^{27}$ Lemoine, F.G., and 14 coauthors, The Development of the Joint NASA GSFC and the National Imagery and Mapping Agency (NIMA) Geopotential Model EGM96, NASA/TP-1998-206861, NASA Goddard Space Flight Center, July 1998, 575 pages.

${ }^{28}$ Cox, C.M., and Chao, B.F., "Detection of a Large-Scale Mass Redistribution in the Terrestrial System Since 1998," Science, 297(5582), Aug. 2, 2002, pp. 831-833.

${ }^{29}$ Marshall, J.A.M., and Luthcke, S.B., "Radiative Force Model Performance for TOPEX/Poseidon Precision Orbit Determination," J. Astron. Sci., 45(2), 1994, pp. 229-246.

${ }^{30}$ Hedin, A.E., "The Atmospheric Model in the Region 90 to 2000 km," Advances in Space Research, 8(5-6), 1988, pp. 9-25.

${ }^{31}$ Knocke, P.C., Ries, J.C., and Tapley, B.D., "Earth Radiation Pressure Effects on Satellites," Proceedings of the AIAA/AAS Astrodynamics Conference, Minneapolis, Minnesota, August 15-17, 1988, pp. 577-586.

${ }^{32}$ Standish, E.M., Newhall, X X, Williams, J.G. and Folkner, W.F., "JPL Planetary and Lunar Ephemerides, DE403/LE403," JPL Inter-Office Memorandum 314.10-127, Jet Propulsion Laboratory, Pasadena, California, 1995.

${ }^{33}$ Altamimi, Z., Sillard, P., and Boucher, C., "ITRF2000: A New Release of the International Terrestrial Reference Frame for Earth Science Applications," J. Geophys. Res.-solid earth, 107(B10), 2002, doi:10.1029/2001JB000561. 